SOI: $\underline{1.1 / \text { TAS }}$ DOI: $10.15863 /$ TAS International Scientific Journal Theoretical \& Applied Science

p-ISSN: 2308-4944 (print) $\quad$ e-ISSN: 2409-0085 (online)

Year: $2015 \quad$ Issue: $08 \quad$ Volume: 28

Published: $30.08 .2015 \quad \underline{\text { http://T-Science.org }}$
Roman Yurievich Kostyuchenko Associate Professor, Candidate of Pedagogical Sciences Associate professor of Mathematics and Methodology of Teaching Mathematics Chair, Omsk State Pedagogical University kryu@bk.ru

SECTION 21. Pedagogy. Psychology. Innovations in the field of education.

\title{
ALGORITHMIC APPROACH IN TEACHING PUPILS OF SOLVING TRIGONOMETRIC EQUATIONS
}

Abstract: Equations are considerable part of Mathematics as science and curriculum at school. Solving of trigonometric equations is not always simple because of objective and subjective reasons. From our point of view, teaching process how to solve trigonometric equations must be organized step-by-step: practice of solving simple trigonometric equations, then solving trigonometric equations of certain kind, and finally, in connections with accumulated knowledge of trigonometric expressions, solving equations of different types. Suggested article shows necessity of such coherence, reviews examples of trigonometric equations, and summarizes solving methods.

Key words: teaching Mathematics, trigonometry, trigonometric equations, solving of trigonometric equations, algorithmic approach.

Language: Russian

Citation: Kostyuchenko RY (2015) ALGORITHMIC APPROACH IN TEACHING PUPILS OF SOLVING TRIGONOMETRIC EQUATIONS. ISJ Theoretical \& Applied Science 08 (28): 80-85.

Soi: http://s-o-i.org/1.1/TAS-08-28-13 Doi: crossef http://dx.doi.org/10.15863/TAS.2015.08.28.13

\section{АЛГОРИТМИЧЕСКИЙ ПОДХОД К ОБУЧЕНИЮ ШКОЛЬНИКОВ РЕШЕНИЮ ТРИГОНОМЕТРИЧЕСКИХ УРАВНЕНИЙ}

Аннотация: Уравнения составляют значимую часть математики как науки, так и школьного курса математики. Решение тригонометрических уравнений не всегда является достаточно простым, этому есть как объективные, так и субъективные причины. На наш взгляд, обучение решению тригонометрических уравнений должно строится поэтапно: отработка навыка решения простейших тригонометрических уравнений, затем решение тригонометрических уравнений определенных видов, и далее, в связи с обогащением знаний о преобразованиях тригонометрических выражений, решение различных типов уравнений. В предлагаемой статье приводится обоснование такой последовательности, рассматриваются примеры тригонометрических уравнений, обобщаются методы их решения.

Ключевые слова: обучение математике, тригонометрия, тригонометрические уравнения, решение тригонометрических уравнений, алгоритмический подход.

Тригонометрический материал составляет значимую часть математики. Его изучение связано с изучением всех разделов школьного курса математики. Действительно, геометрия посредством тригонометрии обогащается количественными отношениями между сторонами и углами треугольника, новой единицей измерения углов - радианы; в началах математического анализа тригонометрия открывает для изучения новый вид функций трансцендентных, дает яркий пример периодических функций; в алгебре расширяются представления учащихся о тождественных преобразованиях, решаются новые виды уравнений. О тригонометрических уравнениях и пойдет речь в данной статье.

Уравнения вообще, и тригонометрические в частности, способствуют формированию у учащихся взглядов на математику как единую научную область, формируют представления о ее целостности, показывают взаимосвязь с реальной действительностью, а также решение уравнений развивает логическое мышление школьников, активизирует творческий потенциал учащихся.

При обучении школьников решению тригонометрических уравнений в контексте алгоритмического подхода следует: 
1) дать определение тригонометрического уравнения;

2) выделить основные типы уравнений и способы их решения;

3) рассмотреть способы решения более сложных уравнений.

Заметим, что многие школьные учебники не дают определение тригонометрическому уравнению вообще. И это во многом оправдано, ибо общее понятие тригонометрического уравнения не входит в логические связи при его решении, а уравнения определенных видов, как правило, определяются. Так, например, в учебнике простейшее тригонометрическое уравнение определяется как «уравнение $f(x)=a$, где $a$ - данное число, а $f(x)$ - одна из основных тригонометрических функций». [2, с. 295]. Как показывает практика, этого определения вполне достаточно для дальнейшего изучения темы.

В соответствии с видом и методами решения тригонометрических уравнений, на наш взгляд, следует выделять три их группы.

I группа - это простейшие тригонометрические уравнения (и сводящиеся к ним уравнения вида $\sin (k x+b)=a$, $\cos (k x+b)=a, \operatorname{tg}(k x+b)=a)$.

Обобщение решения простейших тригонометрических уравнений может быть представлено в виде таблицы (табл. 1):

\section{Решение простейших тригонометрических уравнений.}

\begin{tabular}{|c|c|}
\hline $\begin{array}{l}\sin x=a \\
x=(-1)^{n} \arcsin a+\pi n, n \in Z \quad(-1 \leq a \leq 1) \\
\arcsin (-a)=\arcsin a\end{array}$ & \begin{tabular}{l}
\multicolumn{2}{c}{$\cos x=a$} \\
$x= \pm \arccos a+2 \pi n, n \in Z \quad(-1 \leq a \leq 1)$ \\
$\arccos (-a)=\pi-\arccos a$
\end{tabular} \\
\hline $\begin{array}{l}\sin x=0, \quad x=\pi n, n \in Z \\
\sin x=1, \quad x=\frac{\pi}{2}+2 \pi n, n \in Z \\
\sin x=-1, \quad x=-\frac{\pi}{2}+2 \pi n, n \in Z\end{array}$ & $\begin{array}{l}\cos x=0, \quad x=\frac{\pi}{2}+\pi n, n \in Z \\
\cos x=1, \quad x=2 \pi n, n \in Z \\
\cos x=-1, \quad x=\pi+2 \pi n, n \in Z\end{array}$ \\
\hline $\begin{array}{l}x=\operatorname{arctg} a+\pi n, n \in Z \\
\operatorname{tg} x=a \\
\operatorname{arctg}(-a)=-\operatorname{arctg} a\end{array}$ & $\begin{array}{l}\operatorname{ctg} x=a \\
x=\operatorname{arcctg} a+\pi n, n \in Z \\
\operatorname{arcctg}(-a)=\pi-\operatorname{arcctg} a\end{array}$ \\
\hline
\end{tabular}

Вполне понятно, что каждая из представленных в таблице (см. табл. 1) формул достаточна сложна для школьников, изучающих ее на начальном этапе. Это во многом связано с тем, что здесь «учащиеся впервые имеют дело с бесконечным множеством корней уравнения» $[6$, c. 41]. Поэтому должна быть организована соответствующая работа по осознанному получению, запоминанию и применению данных формул. Например, в названной выше статье [6] автор предлагает свой подход, основанный на графическом решении и учитывающий сложность самих формул и их получения.

II группа - это уравнения определенных видов. Выделим четыре вида.

1. Уравнения, сводящиеся к квадратным заменой $\sin x=t$ или $\cos x=t$ (в более общем случае это уравнения вида $F(f(x))$, где $F-$ рациональная функция, $f(x)$ - одна из основных тригонометрических функций).

\section{Пример 1.}

$2 \cos ^{2} x-3 \cos x+1=0$.

Пусть $\cos x=t$, тогда: $2 t^{2}-3 t+1=0$, $t_{1}=\frac{1}{2}, t_{2}=1$,

1) $\cos x=\frac{1}{2}, x= \pm \frac{\pi}{3}+2 \pi n, n \in Z$;

2) $\cos x=1, x=2 \pi n, n \in Z$.

Ответ : $x= \pm \frac{\pi}{3}+2 \pi n, x=2 \pi n, n \in Z$.

2. Однородные тригонометрические уравнения.

Уравнение вида $a \sin x+b \cos x=0 \quad-$ однородное тригонометрическое уравнение 1-ой степени; $\quad a \sin ^{2} x+b \sin x \cdot \cos x+c \cos ^{2} x=0 \quad-$ однородное тригонометрическое уравнение 2-ой степени, $a \sin ^{3} x+b \sin ^{2} x \cdot \cos x+c \sin x \cdot \cos ^{2} x+d \cos ^{3} x=0$ - однородное тригонометрическое уравнение 3 ей степени и т.д. 
Наиболее распространены в школьном курсе математики однородные уравнения первой и второй степеней. Метод решения таких уравнений - деление на любое слагаемое (как правило, на $\cos ^{2} x$ ), и далее - замена переменной, которая приводит к рациональному уравнению.

Пример 2. (однородное тригонометрическое уравнение 2-ой степени).

$\sin ^{2} x-5 \sin x \cdot \cos x+4 \cos ^{2} x=0$.

Разделим уравнение на $\cos ^{2} x \neq 0$ :

$\frac{\sin ^{2} x}{\cos ^{2} x}-\frac{5 \sin x \cdot \cos x}{\cos ^{2} x}+\frac{4 \cos ^{2} x}{\cos ^{2} x}=0$,

$\operatorname{tg}^{2} x-5 \operatorname{tg} x+4=0$

1) $\operatorname{tg} x=1, x=\frac{\pi}{4}+\pi n, n \in Z$;

2) $\operatorname{tg} x=4, x=\operatorname{arctg} 4+\pi n, n \in Z$.

Значения $x$ при которых $\cos ^{2} x=0$, не удовлетворяют уравнению.

Ответ : $x=\frac{\pi}{4}+\pi n, x=\operatorname{arctg} 4+\pi n, n \in Z$.

Заметим, что данному уравнению не удовлетворяют значения $x$, при которых $\cos ^{2} x=0$ (т.к. в случае $\cos ^{2} x=0$, получаем $\cos x=0$ и из исходного уравнения следует, что $\sin ^{2} x=0$, чего быть не может, поскольку $\sin ^{2} x+\cos ^{2} x=1$ ), поэтому деление обоих частей уравнения на $\cos ^{2} x$ не приводит к потере корней.

Пример 3 (однородное тригонометрическое уравнение 1-ой степени).

$\sin x+\sqrt{3} \cos x=0$.

Разделим уравнение на $\cos x \neq 0$ :

$\operatorname{tg} x+\sqrt{3}=0, \operatorname{tg} x=-\sqrt{3}$,

$x=-\frac{\pi}{3}+\pi n, n \in Z$.

Ответ : $x=-\frac{\pi}{3}+\pi n, n \in Z$.

В рассмотренных двух примерах мы привели решение полных однородных тригонометрических уравнений $(a \neq 0, b \neq 0, c \neq 0)$, однако, если один из этих коэффициентов будет равен нулю, тригонометрическое уравнение обращается в неполное, и решение его требует более сложных размышлений.

Покажем на следующем примере методы решения неполных тригонометрических уравнений.

Пример 4.1 (неполное однородное тригонометрическое уравнение).

$\sin x \cdot \cos x-\cos ^{2} x=0$.

Разделим уравнение на $\cos ^{2} x \neq 0$ :

$\operatorname{tg} x-1=0$,

$x=\frac{\pi}{4}+\pi n, n \in Z$.
Уравнению удовлетворяют значения $x$ при которых $\cos ^{2} x=0$, поэтому в ответе будет вторая серия $x=\frac{\pi}{2}+\pi n, n \in Z$.

Ответ : $x=\frac{\pi}{2}+\pi n, x=\frac{\pi}{4}+\pi n, n \in Z$.

Заметим, что деление уравнения на выражение, содержащее переменную, требует особого внимания, поскольку при этом сужается область допустимых значений и может произойти потеря корней.

Приведем еще одно решение рассматриваемого неполного однородного уравнения. Только в данном случае будем делить обе его части не на $\cos ^{2} x$, как в предыдущем случае, а на $\sin ^{2} x$. Предварительно заметим, что значения $x$, при которых $\sin ^{2} x=0$, не удовлетворяют данному уравнению. Поэтому при делении на $\sin ^{2} x$ потери корней не произойдет.

Пример 4.2 (неполное однородное тригонометрическое уравнение).

$\sin x \cdot \cos x-\cos ^{2} x=0$.

Разделим уравнение на $\sin ^{2} x \neq 0$ :

$\operatorname{ctg} x-\operatorname{ctg}^{2} x=0, \operatorname{ctg} x(1-\operatorname{ctg} x)=0$,

1) $\operatorname{ctg} x=0, x=\frac{\pi}{2}+\pi n, n \in Z$;

2) $\operatorname{ctg} x=1, x=\frac{\pi}{4}+\pi n, n \in Z$.

Значения $x$, при которых $\sin ^{2} x=0$, не удовлетворяют уравнению.

Ответ : $x=\frac{\pi}{2}+\pi n, x=\frac{\pi}{4}+\pi n, n \in Z$.

Отметим, что решение неполного однородного уравнения делением на одно из слагаемых требует от учащихся хорошей математической подготовки. Как показывает опыт, подобные уравнения целесообразнее решать разложением на множители, вынося общий множитель за скобки.

Пример 4.3 (неполное однородное тригонометрическое уравнение).

$\sin x \cdot \cos x-\cos ^{2} x=0$.

Вынесем $\cos x$ за скобки:

$\cos x \cdot(\sin x-\cos x)=0$,

1) $\cos x=0, x=\frac{\pi}{2}+\pi n, n \in Z$,

2) $\sin x-\cos x=0, \operatorname{tg} x-1=0, x=\frac{\pi}{4}+\pi n, n \in Z$.

Ответ : $x=\frac{\pi}{2}+\pi n, x=\frac{\pi}{4}+\pi n, n \in Z$.

3. Уравнения вида $a \sin x+b \cos x=c$.

Уравнения данного вида решаются разными способами. Наиболее распространенные из них: a) сведение к однородному уравнению, 


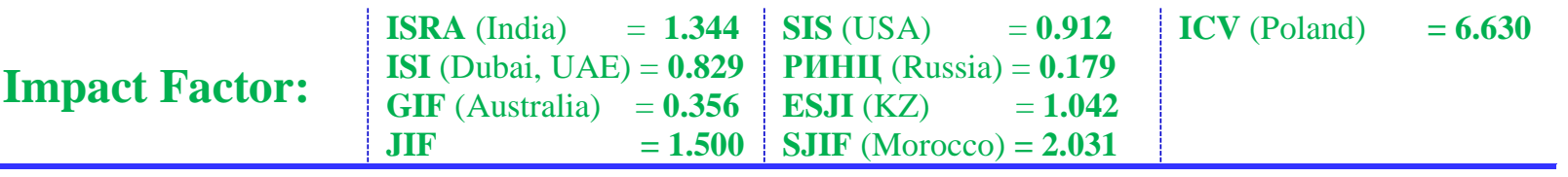

б) введение вспомогательного аргумента, в) решение уравнения с помощью универсальной тригонометрической подстановки.

Решая уравнение посредством сведения его к однородному уравнению, используют тригонометрические формулы двойного угла $\sin x=2 \sin \frac{x}{2} \cdot \cos \frac{x}{2} \quad$ и $\quad \cos x=\cos ^{2} \frac{x}{2}-\sin ^{2} \frac{x}{2}$, постоянную $\quad c \quad$ заменяют через тригонометрические функции, используя основное тригонометрическое тождество $c=c \cdot 1=c \cdot\left(\sin ^{2} \frac{x}{2}+\cos ^{2} \frac{x}{2}\right)=c \cdot \sin ^{2} \frac{x}{2}+c \cdot \cos ^{2} \frac{x}{2}$. В итоге получается однородное тригонометрическое уравнение второй степени, которое решается по известному алгоритму. Пример 5.

$2 \sin x+\cos x=2$.

Преобразуем левую и правую части уравнения

$2 \cdot 2 \sin \frac{x}{2} \cdot \cos \frac{x}{2}+\left(\cos ^{2} \frac{x}{2}-\sin ^{2} \frac{x}{2}\right)=2 \cdot\left(\sin ^{2} \frac{x}{2}+\cos ^{2} \frac{x}{2}\right)$

$3 \sin ^{2} \frac{x}{2}-4 \cdot \sin \frac{x}{2} \cdot \cos \frac{x}{2}+\cos ^{2} \frac{x}{2}=0, \quad \mid: \cos ^{2} \frac{x}{2} \neq 0$

$3 \operatorname{tg}^{2} \frac{x}{2}-4 \operatorname{tg} \frac{x}{2}+1=0$,

1) $\operatorname{tg} \frac{x}{2}=1, \frac{x}{2}=\frac{\pi}{4}+\pi n, n \in Z, x=\frac{\pi}{2}+2 \pi n, n \in Z$;

2) $\operatorname{tg} \frac{x}{2}=\frac{1}{3}, \quad \quad \frac{x}{2}=\operatorname{arctg} \frac{1}{3}+\pi n, n \in Z$,

$x=2 \operatorname{arctg} \frac{1}{3}+2 \pi n, n \in Z$.

Ответ : $x=\frac{\pi}{2}+2 \pi n, x=2 \operatorname{arctg} \frac{1}{3}+2 \pi n, n \in Z$.

Второй способ решения уравнений вида $a \sin x+b \cos x=c$ основан на преобразовании уравнения

К

виду

$\frac{a}{\sqrt{a^{2}+b^{2}}} \sin x+\frac{b}{\sqrt{a^{2}+b^{2}}} \cos x=\frac{c}{\sqrt{a^{2}+b^{2}}}, \quad$ что дает возможность обозначить коэффициенты $\frac{a}{\sqrt{a^{2}+b^{2}}}$ и $\frac{b}{\sqrt{a^{2}+b^{2}}}$ через синус и косинус одного и того же угла, то есть через $\sin \varphi$ и $\cos \varphi$ (в этом случае $\varphi$ называют вспомогательным аргументом); далее по формулам сложения уравнение приводится к виду $\sin (x \pm \varphi)=\frac{c}{\sqrt{a^{2}+b^{2}}}$ или $\cos (x \pm \varphi)=\frac{c}{\sqrt{a^{2}+b^{2}}}$, которые решаются по известному алгоритму.

Пример 6.

$2 \sin x+\cos x=2, \quad \mid: \sqrt{2^{2}+1^{2}}=\sqrt{5}$ $\frac{2}{\sqrt{5}} \sin x+\frac{1}{\sqrt{5}} \cos x=\frac{2}{\sqrt{5}}$,

Пусть $\sin \varphi=\frac{2}{\sqrt{5}}, \cos \varphi=\frac{1}{\sqrt{5}}$, тогда:

$\sin \varphi \sin x+\cos \varphi \cos x=\frac{2}{\sqrt{5}}, \quad \cos (x-\varphi)=\frac{2}{\sqrt{5}}$,

$x-\varphi= \pm \arccos \frac{2}{\sqrt{5}}+2 \pi n, n \in Z$,

$x=\operatorname{arctg} 2 \pm \arccos \frac{2}{\sqrt{5}}+2 \pi n, n \in Z$.

Ответ : $x=\operatorname{arctg} 2 \pm \arccos \frac{2}{\sqrt{5}}+2 \pi n, n \in Z$.

Третий способ решения уравнений вида $a \sin x+b \cos x=c$ основан на приведении этого тригонометрического уравнения к рациональному с помощью универсальной тригонометрической подстановки $\operatorname{tg} \frac{x}{2}=t$.

Пусть $\operatorname{tg} \frac{x}{2}=t$, тогда:

$\sin x=\frac{\sin x}{1}=\frac{2 \sin \frac{x}{2} \cdot \cos \frac{x}{2}}{\cos ^{2} \frac{x}{2}+\sin ^{2} \frac{x}{2}}=\frac{2 \operatorname{tg} \frac{x}{2}}{1+\operatorname{tg}^{2} \frac{x}{2}}=\frac{2 t}{1+t^{2}}$,
$\cos x=\frac{\cos x}{1}=\frac{\cos ^{2} \frac{x}{2}-\sin ^{2} \frac{x}{2}}{\cos ^{2} \frac{x}{2}+\sin ^{2} \frac{x}{2}}=\frac{1-\operatorname{tg}^{2} \frac{x}{2}}{1+\operatorname{tg}^{2} \frac{x}{2}}=\frac{1-t^{2}}{1+t^{2}}$.

Заметим, что применение данной подстановки сужает ОДЗ уравнения, поскольку $\operatorname{tg} \frac{x}{2}$ определен для всех $x$, кроме $x=\pi+2 \pi n, n \in Z$, поэтому нужно проверить, не являются ли числа вида $x=\pi+2 \pi n, n \in Z$ решениями данного уравнения.

Пример 7.

$2 \sin x+\cos x=2$.

Пусть $\operatorname{tg} \frac{x}{2}=t$, тогда $\sin x=\frac{2 t}{1+t^{2}}, \cos x=\frac{1-t^{2}}{1+t^{2}}$, следовательно:

$2 \cdot \frac{2 t}{1+t^{2}}+\frac{1-t^{2}}{1+t^{2}}=2$

$3 t^{2}-4 t+1=0, \quad t_{1}=1, t_{2}=\frac{1}{3}$,

1) $\operatorname{tg} \frac{x}{2}=1, x=\frac{\pi}{2}+2 \pi n, n \in Z$;

2) $\operatorname{tg} \frac{x}{2}=\frac{1}{3}, x=2 \operatorname{arctg} \frac{1}{3}+2 \pi n, n \in Z$.

Проверка показывает, что числа $x=\pi+2 \pi n, n \in Z$, не удовлетворяют заданному уравнению. 
Ответ : $x=\frac{\pi}{2}+2 \pi n, x=2 \operatorname{arctg} \frac{1}{3}+2 \pi n, n \in Z$.

Резюмируя описание решения уравнения вида $a \sin x+b \cos x=c, \quad$ отметим, что применение разных способов к решению одного и того же конкретного уравнения может дать правильные, но совершенно различные по форме ответы. Это наглядно демонстрирует В.А. Далингер [4] при решении уравнения $2 \sin x-2 \cos x=1-\sqrt{3}$.

$$
4 .
$$$$
\text { Уравнения }
$$

вида

$$
a(\sin x \pm \cos x)+b \sin 2 x+c=0 .
$$

Если ввести подстановку $\sin x \pm \cos x=t$, то при возведении ее в квадрат получим:

$(\sin x \pm \cos x)^{2}=t^{2}$,

$\sin ^{2} x \pm 2 \sin x \cdot \cos x+\cos ^{2} x=t^{2}$,

$\left(\sin ^{2} x+\cos ^{2} x\right) \pm 2 \sin x \cdot \cos x=t^{2}$,

$1 \pm \sin 2 x=t^{2}$

$\sin 2 x= \pm\left(t^{2}-1\right)$.

Следовательно, данное трансцендентное тригонометрическое уравнение с помощью указанной подстановки приводится к алгебраическому рациональному уравнению $a \cdot t \pm b\left(t^{2}-1\right)+c=0$.

Пример 8.

$\sin 2 x-4(\sin x+\cos x)+4=0$.

Пусть $\sin x+\cos x=t, \quad$ тогда $\sin 2 x=t^{2}-1$, следовательно $\left(t^{2}-1\right)-4 \cdot t+4=0$,

$t^{2}-4 t+3=0$,

$t_{1}=1, t_{2}=3$,

1) $\sin x+\cos x=1$,

$$
\begin{aligned}
& \frac{1}{\sqrt{2}} \sin x+\frac{1}{\sqrt{2}} \cos x=\frac{1}{\sqrt{2}}, \\
& \sin \frac{\pi}{4} \sin x+\cos \frac{\pi}{4} \cos x=\frac{\sqrt{2}}{2}, \\
& \cos \left(x-\frac{\pi}{4}\right)=\frac{\sqrt{2}}{2}, \\
& x=\frac{\pi}{4} \pm \frac{\pi}{4}+2 \pi n, n \in Z
\end{aligned}
$$

2) $\sin x+\cos x=3$,

$$
x \in \varnothing, m . \kappa \cdot \sin x \leq 1, \cos x \leq 1 \Rightarrow \sin x+\cos x \leq 2 .
$$

Ответ : $x=\frac{\pi}{4} \pm \frac{\pi}{4}+2 \pi n, n \in Z$.

Подводя промежуточный итог, отметим, что нами выделены типовые уравнения, алгоритмы решения которых учащиеся должны понимать и уметь применять в стандартных случаях.

Все же остальные уравнения с помощью тех или иных преобразований сводятся к решению уравнений рассмотренных видов и отнесены нами к третьей группе.

III группа - это уравнения, решение которых предполагает выполнение определенных преобразований, приводящих данное уравнение к решению либо простейших уравнений (І группа), либо уравнений определенных видов (II группа).

Среди уравнений данной группы можно выделить уравнения, допускающие понижение порядка, решаемые с помощью применения формул суммы или умножения тригонометрических функций и др. Решение таких уравнений достаточно полно представлено в научно-методической литературе [1; 3; 5]. Также можно выделить и публикации, касающиеся отдельных аспектов в обучении школьников решению тригонометрических уравнений и неравенств. Например, в статье [10] описывается один из этапов решения тригонометрических уравнений, основанный на переходе к рассмотрению уравнений на периоде или полупериоде; в другой статье [12] излагается подход, основанный на сравнении целых чисел по модулю, который можно применять при нахождении объединения или пересечения полученных множеств корней тригонометрического уравнения.

Bce преобразования, выполняемые над уравнениями III группы, можно разделить на общие, применяемые для всех видов уравнений и неравенств (умножение обеих частей на не равное нулю число, перенос слагаемых из одной части в другую и др.), и специальные, основанные на свойствах тригонометрических функций и преобразованиях тригонометрических выражений. Как показывают результаты ЕГЭ, не все учащиеся успешно справляются с решением тригонометрических уравнений Анализ наиболее часто встречающихся ошибок, возможные причины их возникновения, а также рекомендации по предупреждению подобных ошибок в практике учителя математики можно встретить в статье [11].

Приведенные выше в статье примеры решались алгебраическим методом, однако, наряду с ним, выделяют и графический метод решения уравнений. Заметим, что алгебраический и графический методы решения уравнений являются основными.

Графический метод решения уравнений $f(x)=g(x)$ состоит в отыскании абсцисс точек пересечения графиков функций $y=f(x)$ и $y=g(x)$. Заметим, что при данном методе необходимо учитывать следующее: а) использование знака равенства допустимо после аналитической проверки, в противном случае следует применять знак приблизительного равенства; б) для определения числа корней уравнения $f(x)=g(x)$ 
необходимо исследовать поведение функций $y=f(x)$ и $y=g(x)$ на монотонность, а в некоторых случаях - на выпуклость. Поэтому графический метод решения правильнее называть функционально-графическим. Более того, многие уравнения, предполагающие данный метод решения, зачастую могут быть решены и без построения графиков функций. Например, рассмотрим уравнение $\sin ^{2} x+|\sin x|=0$.

Каждое слагаемое в его левой части не меньше 0 , следовательно, их сумма может быть равна 0 только в том случае, когда каждое слагаемое равно нулю, а это числа $x=\pi n, n \in Z$.

Исходя из сказанного, можно говорить и о приоритетных направлениях в методике обучения учащихся решению тригонометрических уравнений:

1. Доведение до уровня навыка решения простейших тригонометрических уравнений.

2. Доведение до уровня умения (умение выполнение действия при активном контроле сознания, навык - автоматизированное умение) решения уравнений определенных видов, а именно: 1) сводящихся заменой к квадратным, 2) однородных, 3) вида $a \sin x+b \cos x=c$, 4) вида $a(\sin x \pm \cos x)+b \sin 2 x+c=0$.

3. Реализация внутрипредметных связей: «направленность на установление связей с остальным содержанием курса математики» [8, с. 269]. В первую очередь, здесь можно выделить функциональную содержательно-методическую линию [9, с. 192], а также линии числовую и тождественных преобразований.

4. Обучение «обобщенному приему решения уравнений» [7, с. 80] за счет обобщения и систематизации методов в решении уравнений различных видов.

В заключение отметим, что в статье мы уделили большее внимание алгоритмическим методам решения уравнений по сравнению функционально-графическим методом. Одной из причин этого служит то, что функциональнографический метод будет более явно проявляться после изучения других видов трансцендентных уравнений, когда учащиеся овладеют большим арсеналом знаний и умений по решению всех изучаемых в школьном курсе уравнений.

\section{References:}

1. (2005) Algebra i nachala analiza: Kniga dlya uchitelya $\mathrm{k}$ "Sborniku zadach po algebre $\mathrm{i}$ nachalam analiza dlya podgotovki i provedeniya itogovoj attestacii za kurs srednej shkoly": 11 klass / S.A.Shestakov, L.I.Zvavich, I.R.Vysockij, A.R.Ryazanovskij, B.P.Pigarev; pod red. S.A.Shestakova, L.I.Zvavicha. Moscow: Vneshsigma, 2005. - 320 p.

2. (2014) Algebra i nachala matematicheskogo analiza. 10 klass: Uchebnik dlya obshcheobrazovatel'nyh organizacij: bazovyj i uglublyonnyj urovni / S.M. Nikol'skij, M.K. Potapov, N.N. Reshetnikov, A.V. Shevkin. Moscov: Prosveshchenie, 2014. - 431 p.

3. Borodulya IT (1989) Trigonometricheskie uravneniya i neravenstva: Kniga dlya uchitelya. - Moscow: Prosveshchenie, 1989. - 239 p.

4. Dalinger VA (2007) Razmyshleniya po povodu odnoj zadachi EGE po matematike // Matematika v shkole. - № 9. - pp. 34-37.

5. Dalinger VA (1996) Vse dlya obespecheniya uspekha na vypusknyh i vstupitel'nyh ehkzamenah po matematike. Vypusk 6. Trigonometricheskie uravneniya, neravenstva i ih sistemy: Uchebnoe posobie. - Omsk: Izd-vo OmGPU, 1996. - 179 p.
6. Dvoryaninov SV (2013) O tradiciyah, perezhitkah i nedomolvkah $\mathrm{v}$ shkol'noj matematike // Matematika v shkole. - 2013. № 5. - pp. 41-46.

7. Episheva OB (2000) Special'naya metodika obucheniya arifmetike, algebre i nachalam analiza v srednej shkole. Kurs lekcij: Ucheb. posobie dlya vuzov. - Tobol'sk: Izd-vo TGPI im. D.I. Mendeleeva, 2000. - 126 s.

8. (2005) Metodika i tekhnologiya obucheniya matematike. Kurs lekcij: Posobie dlya vuzov / pod nauchn. red. N.L. Stefanovoj, N.S. Podhodovoj. - Moscow: Drofa, 2005. -416 p.

9. Mordkovich AG (2005) Besedy s uchitelyami matematiki: Ucheb.-metod. posobie. - Moscow: Izdatel'skij dom «ONIKS 21 vek»: Izd-vo «Mir i Obrazovanie», 2005. - $336 \mathrm{p}$.

10. Chuchaev II, Nesterova TN (2012) Ob odnom prieme resheniya trigonometricheskih uravnenij // Matematika v shkole. - 2012. - № 9. - pp. 31-38.

11. Shashkina MB, Yakimenko MS (2012) Po goryachim sledam EGE 2012 g.: zadanie C1 // Matematika v shkole. - № 9. - pp. 11-18.

12. Shishilina SM (2011) Sravnenie chisel po modulyu $\mathrm{v}$ trigonometrii // Matematika v shkole. - 2011. - № 1. - pp. 42-45. 